SIMULATED INTERACTIVE MANAGEMENT SERIES

\title{
Simple measures to reduce the rate of contamination of blood cultures in Accident and Emergency
}

\author{
M Madeo, T Jackson, C Williams
}

Emerg Med J 2005;22:810-811. doi: 10.1136/emj.2005.003079

Objectives: To reduce the contamination rate of blood cultures taken in the Accident and Emergency (A\&E) department.

Methods: The standard blood culture sampling kit was supplemented with an instruction sheet on the optimal method for drawing blood cultures and a large $62 \%$ ethyl alcohol impregnated wipe.

Results: There was a statistically significant reduction in the number of contaminants $(p=0.03)$.

Conclusions: Simple measures to encourage skin disinfection and appropriate sampling technique will reduce the incidence of contamination of blood cultures in the A\&E department.

$\mathrm{T}$ e usefulness of blood cultures taken in the Accident and Emergency department (A\&E) remains open to question. In two A\&E based studies only $1.6 \%$ of blood cultures taken in one study had an impact on the management of patients $^{1}$ and in another only $0.52 \%$ potentially had their medical management affected by the positive blood culture results. $^{2}$

Contamination of blood cultures is also a common problem and false positive results due to contaminants may lead to errors both in clinical interpretation and administration of inappropriate treatment. There are several problems in A\&E which may contribute to high contamination rates of blood cultures-these include a rapid turnover of staff, lack of ongoing training, workload, and the nature of the presenting patients. Our study shows the effect that the provision of information on skin decontamination has on the contamination rate in A\&E.

\section{METHODS}

The infection control team, in partnership with the A\&E consultant staff, introduced a blood culture sampling kit. This contained BacT/ALERT (Biomerieux, Basingstoke, Hants, UK) sampling bottles but the bottles were placed in a polythene sleeve with enclosed pocket size instructions on how to take blood cultures (fig 1) and a large 62\% ethyl alcohol impregnated wipe (Purell, Gojo Industries, Arkon, OH, USA) for cleaning the patient's skin before venepuncture.

The medical and nursing staff in A\&E were instructed on how to use the blood culture kits but no additional formal training was given on venepuncture. The interventions were timed to occur in the middle of the junior doctors' rotation period to minimise the effect that staff changes may have had on the study.

The request form was marked in order to allow the laboratory to identify which blood culture samples had been collected using the kit method. Bacteria were identified using standard microbiological techniques. A blood culture contaminant was defined as a usual skin organism that was isolated from only one set of blood cultures in a patient with no evidence of an infection with that organism.

\section{RESULTS}

In the month before the intervention 50 sets of blood cultures were taken, $35(70 \%)$ had no bacterial growth, three $(6 \%)$ were judged to have significant growth, and $12(24 \%)$ were judged to contain contaminants. In the month following the intervention 50 sets of blood cultures were also taken; 37 $(74 \%)$ had no bacterial growth, nine $(18 \%)$ were judged to have significant growth, and four $(8 \%)$ were judged to contain contaminants. There was no statistically significant difference in overall bacterial growth following intervention, $\chi_{(\mathrm{df}=1)}^{2}=2.31, \mathrm{p}=0.13$. There was however a statistically significant reduction in the number of contaminants, $\chi^{2}$ $(\mathrm{df}=2)=7.06, \mathrm{p}=0.03$.

The results are summarised in table 1 and bacterial isolates listed in table 2 .

\section{DISCUSSION}

The role of blood cultures in A\&E remains open to question. However a reduction in the number of contaminants will optimise any relevance that a positive blood culture has to patient management. Most false positive blood cultures are caused by endogenous microbial skin flora so strict skin preparation and good venepuncture technique are important factors in reducing the rate of contamination. ${ }^{34}$ The most common bacterial contaminant identified was coagulase negative staphylococci (CNS); 12 CNS were identified in the month prior to the intervention but only three in the subsequent month.

Until the 1970s CNS were considered almost entirely to be contaminants arising from the skin flora. ${ }^{5}$ It is now recognised that CNS bacteraemia may be associated with the use of indwelling devices such as central venous or haemodialysis catheters or other prosthetic implants. In an earlier study when CNS were isolated in the first 48 hours of hospitalisation, an intravascular device was more frequently associated with episodes of true bacteraemia than in those considered as contamination (7 of 7 (100\%) v 10 of 57 (18\%), respectively; $\mathrm{p}<0.001) .{ }^{6}$ In the absence of such devices we have considered CNS to be contaminants.

Other studies have attempted to identify ways of reducing blood culture contamination rates. A study in the USA associated the use of a dedicated phlebotomy service $(p=0.039)$, use of tincture of iodine for skin disinfection $(p=0.036)$, and application of an antiseptic to the top of the collection device before inoculation $(p=0.018)$ with significantly lower contamination rates. Teaching institutions and a high rate of bed occupancy were demographic factors associated with higher blood culture contamination rates for inpatients. The type of blood culture method used,

Abbreviations: CNS, coagulase negative staphylococci. 
- Withdraw $20 \mathrm{ml}$ blood (5 $\mathrm{ml}$ from pre-teen children, 1-2 $\mathrm{ml}$ in neonates) and inoculate $10 \mathrm{ml}$ into anaerobic bottle first then the remainder into the aerobic bottle.

- Do not change needles between venepuncture and inoculation of the bottles - reduces the risk of contamination and injury.

- Dispose of needle and syringe as a single unit in a sharps container

- Send the samples to the laboratory for incubation ASAP. PATIENTS CURRENTLY RECEIVING ANTIBIOTICS ONLY 5 ML OF BLOOD SHOULD BE INOCULATED PER BOTTLE

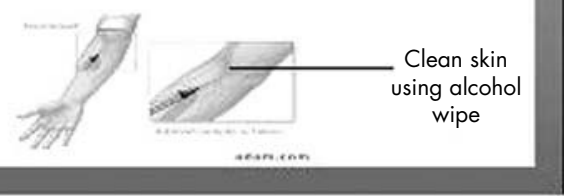

Figure 1 Leaflet enclosed with each set of blood culture bottles.

Table 1 Results of blood cultures taken before and after the intervention

\begin{tabular}{lllll}
\hline & \multicolumn{3}{c}{$\begin{array}{c}\text { Total blood } \\
\text { culture sets }(\%)\end{array}$} & \multicolumn{3}{c}{ No bacterial Significant bacterial Contaminated } \\
growth (\%) & growth $(\%)$ & $(\%)$ \\
\hline Pre $50(100)$ & $35(70)$ & $3(6)$ & $12(24)$ \\
Post $50(100)$ & $37(74)$ & $9(18)$ & $4(8)$ \\
\hline
\end{tabular}

Table 2 Microorganisms recovered from the blood cultures

\begin{tabular}{llllll}
\hline & \multicolumn{2}{l}{ Significant growth } & & \multicolumn{2}{c}{ Contaminants } \\
\cline { 2 - 3 } \cline { 5 - 6 } Microorganisms & Pre & Post & & Pre & Post \\
\hline Staphylococcus epidermidis & 0 & 0 & & 12 & 3 \\
Diptheroids & 0 & 0 & & 0 & 1 \\
Staphylococcus aureus & 0 & 3 & & 0 & 0 \\
Eschericia coli & 1 & 5 & & 0 & 0 \\
Streptococcus spp & 1 & 1 & & 0 & 0 \\
Klebsiella spp & 1 & 0 & & 0 & 0 \\
Total & 3 & 9 & & 12 & 4 \\
\hline
\end{tabular}

specimen volume, or use of a double needle collection procedure did not significantly affect contamination rates. ${ }^{7}$

Studies of disinfectants used for skin preparation have shown conflicting results. One study showed that skin cleaning with chlorhexidine reduced the incidence of blood culture contamination more than povidone-iodine $(1.4 \%$ compared with $3.3 \%, \mathrm{p}=0.004) .{ }^{8}$ But a more recent study comparing $10 \%$ povidone-iodine, $70 \%$ isopropyl alcohol, tincture of iodine, or povidone-iodine with $70 \%$ ethyl alcohol failed to show any significant differences in the blood culture contamination rates between these four antiseptics. ${ }^{9}$

It is likely that the choice of antiseptic makes little difference and the important factor is the use of any skin

\section{PREPARATION FOR TAKING BLOOD-CULTURES}

\section{EQUIPMENT}

$20 \mathrm{ml}$ sterile syringe with 21 gauge needle

Blood-culture bottles (purple top $=$ anaerobic and blue top $=$ aerobic)

For babies a single paediatric bottle with a yellow top should be used.

Skin disinfectant

Sharps container

Sterile/Non-sterile gloves

\section{PROCEDURE}

- Select two Bact/Alert bottles (one purple and one blue)

- Label each bottle with patient's name, DOB, unit number, date and time of collection - both on the specimen and on the laboratory request form.

DO NOT PLACE LABELS ON BOTTLE

- Clean the skin by swabbing concentrically using enclosed alcohol wipe from the venepuncture site outwards for a minimum of 30 seconds - allow the site to dry completely before performing venepuncture (1-2 minutes)

- Do not touch prepared skin with unglove finger

Disinfect the tops of Bact/Alert bottles using 70\% alcohol (do not use iodine)

preparation. We have found that the inclusion of an appropriate skin wipe and instruction sheet has significantly reduced the rate of contamination of blood cultures taken in our Accident and Emergency department.

\section{Authors' affiliations}

M Madeo, C Williams, Department of Infection Control, Hull and East Yorkshire Hospitals, Hull, UK

T Jackson, Department of Accident and Emergency, Hull and East Yorkshire Hospitals, Hull, UK

Correspondence to: Dr C Williams, Department of Microbiology, Yorkhill Hospital, Dalnair Street, Glasgow G3 8SJ, UK; craig.williams@yorkhill.scot.nhs.uk

\section{REFERENCES}

1 Kelly AM. Clinical impact of blood cultures taken in the emergency department. J Accid Emerg Med 1998;15:254-6.

2 Sturmann KM, Bopp J, Molinari D, et al. Blood cultures in adult patients released from an urban emergency department: a 15-month experience. Acad Emerg Med 1996;3:768-75.

3 Trautner WB, Clarridge EJ, Darouiche RO. Skin antisepsis kits containing alcohol and chlorhexidine gluconate or tincture of iodine are associated with low rates of blood culture contamination. Infect Control Hosp Epidemiol 2002;7:397-401

4 Weinstein MP. Current blood culture methods and systems: clinical concepts, technology, and interpretation of results. Clin Infect Dis 1996;23:40-46.

5 McGowan JE. Changing etiology of nosocomial bacteremia and fungemia and other hospital-acquired infections. Rev Infect Dis 1985;7(Suppl 3):S357-370.

6 Finkelstein R, Fusman R, Oren I, et al. Clinical and epidemiologic significance of coagulase-negative staphylococci bacteremia in a tertiary care university Israeli hospital. Am J Infect Control 2002;1:21-5.

7 Schifman RB, Strand CL, Meier FA, et al. Blood culture contamination: a College of American Pathologists Q-Probes study involving 640 institutions and 497134 specimens from adult patients. Arch Pathol Lab Med $1998 ; 122: 216-21$.

8 Mimoz O, Karim A, Mercat A, et al. Chlorhexidine compared with povidoneiodine as skin preparation before blood culture. A randomized, controlled trial. Ann Intern Med 1999;131:834-7.

9 Calfee DP, Farr BM. Comparison of four antiseptic preparations for skin in the prevention of contamination of percutaneously drawn blood cultures: a randomized trial. J Clin Microbiol 2002;5:1660-5. 